\title{
Estimates Away From a Discontinuity for Dissipative Galerkin Methods for Hyperbolic Equations
}

\author{
By William J. Layton*
}

\begin{abstract}
We consider the approximate solution of the initial value problem$$
\frac{\partial u}{\partial t}=\frac{\partial u}{\partial x}, \quad u(x, 0)=v(x)
$$

by a dissipative Galerkin method. When $v$ is taken to have a jump discontinuity at zero, that discontinuity will propagate along $x+t=0$, in the true solution $u$. Estimates in $L_{2}$ and $L_{\infty}$ of the pollution effects of the discontinuity are found. These estimates show those effects to decay exponentially in $h^{-1}$ in regions a fixed distance $d$ from the discontinuity and exponentially in $d$ for fixed $h$.
\end{abstract}

I. Introduction. In this paper the question of approximating, by Galerkin methods, discontinuous solutions to the initial value problem

$$
\frac{\partial u}{\partial t}=\frac{\partial u}{\partial x}, \quad u(x, 0)=v(x),
$$

is considered. Here $v$ is taken to be an $L_{2}(-\infty, \infty)$ function, smooth, except for a jump discontinuity at $x=0$. This discontinuity will propagate along the characteristic $x+t=0$ of the true solution $u(x, t)=v(x+t)$ of (1).

In the usual, continuous in time, Galerkin method for (1) a subspace $S_{h}$ of $H^{1}(-\infty, \infty)$ is chosen and the approximate solution is computed as a differentiable $\operatorname{map} u^{h}:[0, T] \rightarrow S_{h}$ by

$$
\begin{aligned}
\left(\frac{\partial u^{h}}{\partial t}, \phi\right) & =\left(D u^{h}, \phi\right) \text { for } \phi \in S_{h}, D=\frac{\partial}{\partial x}, \\
u^{h}(0) & \in S_{h}, \quad \text { an approximation to } v,
\end{aligned}
$$

where $(\cdot, \cdot)$ denotes the usual inner product on $L_{2}(-\infty, \infty)$.

The usual Galerkin method is not appropriate for the solution of (1) with $v(x)$ as described for several reasons:

(i) The rate of convergence of the method (2), (3) depends upon the global smoothness of $u$. Here $u$ is piecewise $C^{\infty}$ but globally only $L_{2}$.

(ii) The usual Galerkin method is a strictly conservative method with an infinite domain of dependence. Experience with such methods indicates that the approximate solution will be hopelessly polluted by the discontinuity in $u$ even far away from that discontinuity.

Received December 21, 1979; revised May 2, 1980.

1980 Mathematics Subject Classification. Primary 65M15; Secondary 65N30.

*Work partially supported by the U. S. Army Research Office. 
For these reasons a dissipative Galerkin method will be considered for the problem (1). Estimates of the error outside a neighborhood of the discontinuity will be derived for this method. These estimates imply, for example, that the error for (1), outside a neighborhood of $x+t=0$, has two parts, the first being the error applied to smooth solutions of (1). The second component is the pollution effect of the discontinuity. This part is shown to decay exponentially in $h^{-1}$ for regions a fixed distance $d$ from the shock front and exponentially in $d$ for fixed $h$.

II. The Method. We shall choose as $S_{h}$ the space of splines of order $\mu$ of at most power growth. Define, following, e.g., Schoenberg [11] and Thomé [13], the $B$ splines of order $\mu \geqslant 2$. Let $\chi$ be the characteristic function of $\left[-\frac{1}{2}, \frac{1}{2}\right]$. For $\mu \geqslant 2$ define $\phi$ and $\phi_{l}$ by $\phi=\chi^{* \mu}, \phi_{l}(x)=\phi\left(h^{-1} x-l\right) . S_{h}$ is taken to be the space of splines of at most power growth

$$
S_{h}=\left\{\sum_{l} C_{l} \phi_{l}: C_{l}=O\left(|l|^{q}\right) \text { as }|l| \rightarrow \infty \text { for some } q\right\} \text {. }
$$

We shall consider a nonstandard Galerkin method for the problem (1). This method is similar to a method first proposed by Dendy [5] and analyzed by Wahlbin, [17] and [18], who proved convergence for smooth solutions of some linear and nonlinear hyperbolic equations. This method adds dissipation to the usual method by use of a nonstandard variational principle.

We shall seek $u^{h}:[0, T] \rightarrow S_{h}$, as a differential map satisfying

$$
\begin{gathered}
\left(\frac{\partial u^{h}}{\partial t}-D u^{h}, \phi_{l}-h D \phi_{l}\right)=0, \quad l \in \mathbf{Z}, \\
u^{h}(0)=I_{h} v \in S_{h} .
\end{gathered}
$$

By $I_{h}$ in (5) we denote the spline interpolation operator, that is, $I_{h} v$ is that element of $S_{h}$ satisfying $\left(I_{h} v\right)(j h)=v(j h), j \in \mathbf{Z}$.

Next some results concerning the method (4), (5) shall be collected. Using techniques developed by Thomée [13] and Thomée and Wendroff [15], Wahlbin [17] has shown that the solution operator, $G_{h}(t)$, to the Galerkin equations (4), (5) factors as $G_{h}(t)=I_{h} F_{k}(t)$, where $I_{h}$ is the spline interpolation operator and $F_{k}(t)$ is a finite difference operator.

For $\mu \geqslant 2$ and integer $\sigma, 0 \leqslant \sigma \leqslant 2 \mu-2$, define the trigonometric polynomials

$$
g_{\mu, \sigma}^{(\theta)}=h^{\sigma-1}(-i)^{\sigma-2 \nu} \sum_{l=-\infty}^{\infty}\left(D^{\sigma-\nu} \phi_{0}, D^{\nu} \phi_{l}\right) e^{-i l \theta},
$$

with $\nu=[\sigma / 2]$. Note that the factor $h^{\sigma-1}$ makes $g_{\mu, \sigma}$ independent of $h$. The next proposition collects some useful results of [17] concerning the method (4), (5).

Proposition 1. Let $G_{h}(t)$ be the solution operator to (4), (5). For $\lambda>0$ fixed, $k=\lambda h$, consider $t$ of the form $t=n k$. Then $G_{h}(t)$ factors as

$$
G_{h}(t)=I_{h}\left(F_{k}\right)^{n}, \quad t=n k>0,
$$

where $I_{h}$ is the spline interpolation operator and $F_{k}$ is a finite difference operator with symbol

$$
a(\theta)=\exp \left(\lambda \frac{i g_{\mu, 1}^{(\theta)}-g_{\mu, 2}^{(\theta)}}{g_{\mu, 0}^{(\theta)}+i g_{\mu, 1}^{(\theta)}}\right)=\exp (\lambda P(\theta)),
$$

where $\operatorname{Re} P(\theta) \leqslant-c \theta^{2 \mu}$, for $0 \leqslant|\theta| \leqslant \pi$, and $\operatorname{Im} P(\theta)=\theta\left(1+O\left(\theta^{2 \mu}\right)\right)$ as $\theta \rightarrow 0$. 
Proof. See Wahlbin [17].

From this one obtains that $F_{k}$ is accurate of order $2 \mu-1$, dissipative of order $2 \mu$, and stable in $L_{2}$ and $L_{\infty}$. Further, $L_{2}$-optimality of the error follows; for more details see Wahlbin [17] or [18]:

THEOREM 1. Suppose the approximate solution to the initial value problem (1) is computed using the method (4), (5). Then there is a positive constant $c$ such that, for $v \in H^{\mu}(-\infty, \infty)$,

$$
\max _{0<t<T}\left\|u(t)-u^{h}(t)\right\|_{L_{2}} \leqslant c h^{\mu} \max _{0<t<T}\|u(t)\|_{H^{\mu}}
$$

Proof. See Wahlbin [17] or [18].

In the following, the $l_{2, h}$ norm shall denote

$$
\|v\|_{l_{2, h}}^{2}=h \sum_{j=-\infty}^{\infty} v(j h)^{2} .
$$

Also for $t=n k$ we shall write $\left(F_{k}\right)^{n}$ as $F_{k}(t)$.

III. Discontinuous Initial Data. In this section the problem outlined in the introduction is considered. The initial data is taken to be an $L_{2}$ function vanishing for positive $x$ with a jump discontinuity at zero and smooth on $(-\infty, 0)$. The case of a function smooth on $(-\infty, 0)$ and $(0, \infty)$ with a jump at zero may be reduced to a function vanishing for positive $x$ by subtracting a smooth function agreeing with the initial data on $(0, \infty)$. The analogous work on this question in finite difference theory was carried out in [1], [3], [6], and [12]. These estimates are shown to hold in the finite element case as well. This work depends upon using spline interpolation theory to derive estimates upon the error in interchanging spline interpolation and multiplication by a characteristic function.

To be more specific, the true solution of (1) for such a $v(x)$ vanishes for $x>-t$. Therefore $u^{h}(x, t)$ is estimated in terms of the distance $d$ of $x$, parallel to the $x$-axis, to the shock front $x+t=0$. If $\chi_{y}$ denotes the characteristic function of $(y, \infty)$, we estimate $\left\|\chi_{d-t} u^{h}(t)\right\|_{L_{2}}$ and $\left\|\chi_{d-t} u^{h}(t)\right\|_{L_{\infty}}$. The basic result is that these two quantities decay exponentially in $d$ for fixed $h$ and exponentially in $h^{-1}$ for fixed $d$. An analogous result can be shown for functions $v(x)$ vanishing on the other side of the shock front. These results, with Theorem 1, are then combined to give the result announced in the introduction.

THEOREM 2. Suppose the approximate solution of (1) is computed using method (4), (5) with $\mu$ even. Suppose also $v(x) \in l_{2, h} \cap L_{2} \cap L_{\infty}$ and that $v$ vanishes for positive $x$. Then there are positive constants $C, c$, and $\alpha(C$ and $\alpha$ depending upon $\mu)$ such that

$$
\left\|x_{d-t} u^{h}(t)\right\|_{L_{2}} \leqslant C\|v\|_{L_{\infty}} h^{1 / 2} \exp \left(-\alpha h^{-1} d\right)+\exp \left(-c n \min \left\{d_{t}^{\kappa}, d_{t}\right\}\right)\|v\|_{l_{2}}
$$

with $t=n k>0, d_{t}=d t^{-1}$. Also $\kappa=2 \mu /(2 \mu-1)$.

TheOREM 3. Suppose the hypotheses of Theorem 2 hold. Suppose also $|x|^{-a} v(x) \in$ $L_{\infty}$ for some $a \geqslant 0$. Then

$$
\begin{aligned}
\left\|\chi_{d-t} u^{h}(t)\right\|_{L_{\infty}} \leqslant & C\|v\|_{L_{\infty}} \exp \left(-\alpha h^{-1} d\right) \\
& +C h^{a / \kappa} \exp \left(-c n \min \left\{d_{t}^{\kappa}, d_{t}\right\}\right)\left\||x|^{-a} v\right\|_{L_{\infty}}
\end{aligned}
$$

holds for $t \leqslant T$, with $d_{t}, C, c, \alpha$, and $\kappa$ as in Theorem 3.1. 
Proof of the Theorems. Note that $\chi_{d-t} u^{h}(t)=\chi_{d-t} I_{h} F_{k}(t) v$. We would like to interchange $I_{h}$ and multiplication by $\chi_{d-t}$. To do so the error in interchanging these two operations must be estimated. To this end, consider the cardinal spline interpolation problem:

For $\mu$ even, we seek $L_{h}(x) \in S_{h}$ satisfying $L_{h}(x) \in L_{\infty}(-\infty, \infty)$ and

$$
L_{h}(j h)= \begin{cases}1, & \text { if } j=0 \\ 0, & \text { if } j \neq 0 .\end{cases}
$$

The following basic result is known about this problem [10], [11].

THEOREM 4. For $\mu$ even, there is a unique $L_{h}$ in $S_{h}$. Furthermore, there are positive constants $A$ and $\alpha$, depending upon $\mu$, such that

$$
\left|L_{h}(x)\right|<A \exp \left(-\alpha h^{-1}|x|\right), \quad x \in \mathbf{R} .
$$

Also, for $C=\left(c_{j}\right) \in l_{\infty}$, the unique function $S_{h}(x)$ in $S_{h}$ satisfying $S_{h}(j h)=c_{j}$, $S_{h}(x) \in L_{\infty}$ is given by

$$
S_{h}(x)=\sum_{j=-\infty}^{\infty} c_{j} L_{h}(x-j h),
$$

the series converging absolutely and uniformly on each compact interval.

This shall be used to produce the desired estimate. For a function $f(x) \in L_{\infty}$, expand $I_{h} f$ and $I_{h}\left(\chi_{y} f\right)$ using Theorem 4:

$$
\begin{aligned}
& \left(I_{h} f\right)(x)=\sum_{j=-\infty}^{\infty} f(j h) L_{h}(x-j h), \\
& I_{h}\left(\chi_{y} f\right)(x)=\sum_{j=J}^{\infty} f(j h) L_{h}(x-j h),
\end{aligned}
$$

where $J=\min \{j: j h>y\}$. Thus,

$$
\begin{aligned}
\left|\left(I_{h} f\right)(x)-I_{h}\left(\chi_{y} f\right)(x)\right| & =\left|\sum_{j=-\infty}^{J-1} f(j h) L_{h}(x-j h)\right| \\
& \leqslant A\|f\|_{L_{\infty}} \sum_{j=-\infty}^{J-1} \exp \left(-\alpha\left|x h^{-1}-j\right|\right) .
\end{aligned}
$$

Setting $y=d / 2-t$ and considering only $x>d-t$ gives

$$
\begin{aligned}
\left|I_{h} f(x)-I_{h}\left(\chi_{d / 2-t} f\right)(x)\right| & \leqslant C\|f\|_{L_{\infty}} \exp \left(-\alpha x h^{-1}\right) \sum_{j=-\infty}^{\infty} \exp (\alpha j) \\
& \leqslant C\|f\|_{L_{\infty}} \exp \left(-\alpha h^{-1}(x-(d / 2-t))\right) .
\end{aligned}
$$

Thus, (8) gives a pointwise estimate and an $L_{2}$ estimate

$$
\begin{gathered}
\left\|I_{h} f-I_{h}\left(\chi_{d / 2-t} f\right)\right\|_{L_{\infty}(d-t, \infty)} \leqslant C\|f\|_{L_{\infty}} \exp \left(-\frac{\alpha}{2} h^{-1} d\right), \\
\left\|I_{h} f-I_{h}\left(\chi_{d / 2-t} f\right)\right\|_{L_{2}(d-t, \infty)} \leqslant C\|f\|_{L_{\infty}} h^{1 / 2} \exp \left(-\frac{\alpha}{2} h^{-1} d\right) .
\end{gathered}
$$

Now return to the problem at hand. For $d>0$ and $p=2, \infty$, there follows

$$
\begin{aligned}
\left\|I_{h} \chi_{d-t} F_{k}(t) v\right\|_{L_{p}(-\infty, \infty)} \leqslant & \left\|I_{h}\left(\chi_{d-t} F_{k}(t) v\right)\right\|_{L_{p}(d-t, \infty)} \\
& +\left\|\chi_{d / 2-t}\left(I_{h} F_{k}(t) v\right)-I_{h}\left(\chi_{d / 2-t} F_{k}(t) v\right)\right\|_{L_{p}(d-t, \infty)} .
\end{aligned}
$$


These two terms shall be estimated separately. Since spline interpolation is a bounded linear operator between $L_{\infty}$ and $L_{\infty}$ and between $L_{2}$ and $l_{2, h}$ (but not $L_{2}$ and $\left.L_{2}\right)[8]$, [11] we have

$$
\begin{aligned}
& \left\|I_{h}\left(\chi_{d-t} F_{k}(t) v\right)\right\|_{L_{2}} \leqslant C\left\|\chi_{d-t} F_{k}(t) v\right\|_{l_{2, h}}, \\
& \left\|I_{h}\left(\chi_{d-t} F_{k}(t) v\right)\right\|_{L_{\infty}} \leqslant C\left\|\chi_{d-t} F_{k}(t) v\right\|_{L_{\infty}} .
\end{aligned}
$$

Finite difference theory shall be used to estimate the above two quantities.

The finite difference problem has been addressed in [1], [3], [6] or [12]. In [3] estimates on $\left\|\chi_{d-t} F_{k}(t) v\right\|_{L_{p}}$ are derived for $p=2$ and $\infty$ using inequalities for the symbol $a(\theta)$ of $F_{k}$ in a neighborhood of the real axis. If $F_{k}(t)$ is considered as a Toeplitz matrix active between $l_{2}$ and $l_{2}$ instead of $L_{2}$ and $L_{2}$, these estimates on $a(\theta)$ may be used to derive $l_{2}$ estimates on $\chi_{d-t} F_{k}(t) v$. Specifically, using the notation of Theorems 1 and 2, there follows

$$
\left\|\chi_{d-t} F_{k}(t) v\right\|_{l_{2, h}} \leqslant \exp \left(-c n \min \left\{d_{t}^{\kappa}, d_{t}\right\}\right)\|v\|_{l_{2, h}} .
$$

The corresponding $L_{\infty}$ estimate in [3] reads

$$
\left\|\chi_{d-t} F_{k}(t) v\right\|_{L_{\infty}} \leqslant C h^{\alpha / \kappa} \exp \left(-c n \min \left\{d_{t}^{\kappa}, d_{t}\right\}\right)\left\||x|^{-a} v\right\|_{L_{\infty}} .
$$

The theorems now follow by setting $f=F_{k}(t) v$ in (9), (10), using the fact that $F_{k}$ is stable in $L_{\infty}$ and using inequalities (9), (10), (11), (12), and (13).

Remarks. A corresponding analysis may be carried out for fully discrete schemes and for other methods of introducing dissipation into the Galerkin method. For an analysis of either case or more details upon the present method see Chapter 3 of [7]. Also, corresponding results may be shown for $\mu$ odd by posing the interpolation operator and the finite difference operator at midpoints rather than at the knots.

Department of Mathematics

University of Tennessee

Knoxville, Tennessee 37916

1. M. Y. T. Apelkrans, "On difference schemes for hyperbolic equations with discontinuous initial values," Math. Comp., v. 22, 1968, pp. 525-539.

2. Ph. Brenner \& V. ThOMÉe, "Stability and convergence rates in $L_{p}$ for certain difference schemes," Math. Scand., v. 27, 1970, pp. 5-23.

3. Ph. Brenner \& V. ThOmée, "Estimates near discontinuities for some difference schemes," Math. Scand., v. 28, 1971, pp. 329-340.

4. A. Calderón, F. Spitzer \& H. Widom, "Inversion of Toeplitz matrices," Illinois J. Math., v. 3, 1959, pp. 490-498.

5. J. E. DENDY, "Two methods of Galerkin type achieving optimum $L_{2}$ rates of convergence for first order hyperbolics," SIAM J. Numer. Anal., v. 11, 1974, pp. 637-653.

6. G. W. Hedstrom, "The rate of convergence of some difference schemes," SIAM J. Numer. Anal., v. 5,1968 , pp. $363-406$.

7. W. J. LAYTON, Ph. D. Thesis, University of Tennessee, 1980.

8. R. RichaRDS, Uniform Spline Interpolation Operators in $L_{2}$, MRC Tech. Report \# 1305, University of Wisconsin, November 1972.

9. R. D. Richtmyer \& K. W. Morton, Difference Methods for Initial Value Problems, 2nd ed., Interscience, New York, 1967.

10. I. J. Schoenberg, “Cardinal interpolation and spline functions. II," J. Approx. Theory, v. 6, 1972, pp. 404-420.

11. I. J. Schoenberg, Cardinal Spline Interpolation, Regional Conference Series in Applied Math., \# 12, SIAM, Philadelphia, Pa., 1973.

12. S. I. Serdyukova, "Oscillations which occur in the numerical computation of the discontinuous solutions of differential equations," Ž. Vycisl. Mat. i Mat. Fiz., v. 11, 1971, pp. 411-424. 
13. V. Тноме́E, "Spline approximation and difference schemes for the heat equation," The Mathematical Foundations of the Finite Element Method with Applications to Partial Differential Equations (A. K. Aziz, Ed.), Academic Press, New York, 1972.

14. V. Tноме́e, "Convergence estimates for semidiscrete Galerkin methods for initial value problems," Numerische, insbesondere approximationstheoretische Behandlung von Funktionalgleichungen, Lecture Notes in Math.,v. 333, Springer, Berlin, 1973.

15. V. Thомée \& B. WendrofF, "Convergence estimates for Galerkin methods for variable coefficient initial value problems," SIAM J. Numer. Anal., v. 11, 1974, pp. 1059-1068.

16. R. S. VARGA, Functional Analysis and Approximation Theory in Numerical Analysis, Regional Convergence Series in Applied Math., \# 3, SIAM, Philadelphia, Pa., 1971.

17. L. B. WahlBin, "A dissipative Galerkin method for the numerical solution of first order hyperbolic equations," Mathematical Aspects of Finite Elements in Partial Differential Equations (C. de Boor, Ed.), Academic Press, New York, 1974.

18. L. B. WAHLBIN, "A dissipative Galerkin method applied to some quasilinear hyperbolic equations," R.A.I.R.O., v. 8, 1974, pp. 109-117.

19. L. B. WAHLBIN, "A modified Galerkin procedure with Hermite cubics for hyperbolic problems," Math. Comp., v. 29, 1975, pp. 978-984. 